\title{
Perfil e tempo de permanência dos usuários admitidos no pronto-socorro adulto de um
} hospital público

\section{Profile and length of stay of users admitted to the adult emergency department of a public hospital}

\section{Perfil y duración de la estancia de los usuarios ingresados en el servicio de urgencias para adultos de un hospital público}

Recebido: 24/09/2020

Aprovado: 02/06/2021

Publicado: 14/10/2021

\section{Cintia Machado Dutra ${ }^{1}$ \\ Lorena Daher ${ }^{2}$ \\ Suzel Regina Ribeiro Chavaglia ${ }^{3}$ \\ Lúcia Aparecida Ferreira ${ }^{4}$ \\ Débora de Oliveira Sacramento 5 \\ Joyce Caroline Lucas Santos ${ }^{6}$}

Trata-se de uma pesquisa transversal com delineamento descritivo e abordagem quantitativa do tipo analítico, realizado em um hospital público de ensino, com dados de prontuários, levando em conta o período de novembro de 2017 a janeiro de 2018, com objetivo de conhecer o perfil e o tempo de permanência dos usuários admitidos no pronto-socorro adulto. Utilizou-se análise estatística descritiva para obtenção de frequência absoluta e relativa e, posteriormente, foi realizado o modelo de regressão logística binária, por meio da análise bruta, que testou as variáveis independentes e sua associação com a variável dependente, adotou-se o valor de $\mathrm{p}<0,20$, para proceder à análise ajustada. Considerou-se 304 sujeitos, em sua maioria $(63,5 \%)$ do sexo masculino, procedentes do atendimento pré-hospitalar do próprio município, com menor grau de prioridade de atendimento e tempo de permanência no pronto-socorro por período superior a 24 horas. Os resultados demonstram que os sujeitos atendidos pela clínica médica referenciados por demanda espontânea tem quatro vezes mais chance de permanecer internados por mais de 24 horas no serviço. Os diagnósticos atribuídos aos sujeitos não se enquadraram nas condições sensíveis à Atenção Primária. Verifica-se a necessidade de identificação das fragilidades da rede de atenção à saúde e da adoção de intervenções para redução do tempo de permanência e da saturação da capacidade operacional.

Descritores: Perfil de saúde; Tempo de internação; Serviços médicos de emergência; Hospitalização.

This is a cross-sectional study with a descriptive design and a quantitative analytical approach, carried out in a public teaching hospital, with data from medical records, taking into account the period from November 2017 to January 2018 , in order to access the profile and the length of stay of users admitted to the adult emergency room. Descriptive statistical analysis was used to obtain absolute and relative frequency and, subsequently, the binary logistic regression model was performed, through crude analysis, which tested the independent variables and their association with the dependent variable, adopting the value of $\mathrm{p}<0.20$, to carry out the adjusted analysis. 304 subjects were considered, mostly ( $63.5 \%)$ male, coming from pre-hospital care in the municipality, with a lower degree of priority of care and length of stay in the emergency room for more than 24 hours. The results demonstrate that subjects seen by the medical clinic who are referred by spontaneous demand are four times more likely to remain hospitalized for more than 24 hours in the service. The diagnoses attributed to the subjects did not fit into the conditions regarding Primary Care. There is a need to identify the weaknesses of the health care network and to adopt interventions to reduce the length of stay and the saturation of operational capacity.

Descriptors: Health profile; Length of stay; Emergency medical services; Hospitalization.

Se trata de una investigación transversal con diseño descriptivo y enfoque cuantitativo de tipo analítico, realizada en un hospital escuela público, con datos procedentes de las historias clínicas, teniendo en cuenta el periodo comprendido entre noviembre de 2017 y enero de 2018, con el objetivo de conocer el perfil y el tiempo de estancia de los usuarios ingresados en el servicio de urgencias para adultos. Se utilizó el análisis estadístico descriptivo para obtener la frecuencia absoluta y relativa y, posteriormente, se realizó el modelo de regresión logística binaria, a través del análisis bruto, que probó las variables independientes y su asociación con la variable dependiente, se adoptó el valor de p $<0,20$, para proceder al análisis ajustado. Se consideraron 304 sujetos, mayoritariamente (63,5\%) de sexo masculino, procedentes de la atención prehospitalaria de la ciudad, con menor prioridad asistencial y duración de la estancia en servicios de urgencias por un periodo superior a 24 horas. Los resultados muestran que los sujetos atendidos por la clínica médica remitidos por demanda espontánea tienen cuatro veces más probabilidades de permanecer hospitalizados más de 24 horas en el servicio. Los diagnósticos atribuidos a los sujetos no se ajustaron a las condiciones sensibles a la Atención Primaria. Es necesario identificar los puntos débiles de la red de atención a la salud y adoptar intervenciones para reducir la duración de la estancia y la saturación de la capacidad operativa.

Descriptores: Perfil de salud; Tiempo de internación; Servicios médicos de urgencia; Hospitalización.

1. Enfermeira. Especialista em Enfermagem em Terapia Intensiva. Mestre em Enfermagem Fundamental. Tutora do Programa de Residência em Enfermagem em Urgência e Trauma da Universidade Federal do Triângulo Mineiro (UFTM). Chefe do Setor de Urgência e Emergência do Hospital de Clínicas (HC) da UFTM - Filial EBSERH. Uberaba, MG, Brasil. ORCID: 0000-0001-5120-6111 E-mail: cintia.dutra@uftm.edu.br

2. Enfermeira. Especialista em Urgência e Emergência, Especialista em Unidade de Terapia Intensiva. Especialista em Enfermagem em Urgência e Trauma. Enfermeira no Pronto Socorro Adulto do Hospital e Maternidade Celso Pierro, Campinas, SP, Brasil. ORCID: 0000-0002-1370-5486 E-mail: lorena_daher@hotmail.com

3. Enfermeira. Especialista em Gestão em Saúde. Mestre, Doutora e Pós Doutora em Enfermagem. Professora Titular do Programa de Pós Graduação em Atenção à Saúde (PPGAS) e do Curso de Graduação em Enfermagem (CGE) da UFTM, Uberaba, MG, Brasil. ORCID: 0000-0001-7033-0185 E-mail: suzel.ribeiro@yahoo.com.br

4. Enfermeira. Especialista em Educação Profissional em Enfermagem. Mestre em Enfermagem Psiquiátrica. Doutora em Enfermagem. Professora Associada do PPGAS e coordenadora do CGE da UFTM Uberaba, MG, Brasil. ORCID: 0000-0001-6469-5444 E-mail: lap2ferreira@yahoo.com.br

5. Enfermeira. Especialista em Enfermagem em Urgência e Trauma. Especializanda em Saúde do Adulto na modalidade Residência Multiprofissional pelo HC/UFTM, Uberaba, MG, Brasil. ORCID: 0000-0001-6448-8330 E-mail: deborasacramento18@gmail.com

6. Enfermeira. Especialista em Urgência e Trauma. Hospital Santa Catarina de Blumenau, SC, Brasil. ORCID: 0000-0003-1150-3255 E-mail: joycelucass@hotmail.com 


\section{INTRODUÇÃo}

A

superlotação e a média de permanência superior a 24 horas nas unidades de prontosocorro, impactam diretamente na qualidade dos serviços, visto que afetam a

rotatividade de leitos, incrementam os custos, contribuem com a ocorrência de eventos adversos e aumentam a sobrecarga de trabalho dos profissionais ${ }^{1}$.

Dentre os aspectos mais questionados quanto aos atendimentos realizados nas unidades de pronto-socorro, destacam-se a demanda espontânea por parte dos usuários e os critérios adotados para o referenciamento a essas unidades. Ainda é comum encontrar nesses ambientes, pessoas em filas por longos períodos, sendo o atendimento realizado por ordem de chegada e não por prioridades clínicas, o que preocupa as autoridades de saúde².

Nesse contexto foi determinada uma associação entre a Política Nacional de Atenção às Urgências e a Política Nacional de Humanização, com vistas a direcionar o usuário para o atendimento de condições agudas em qualquer porta de entrada da rede, sendo estabelecidos critérios de acolhimento baseados na classificação de risco ${ }^{3}$.

Em instituições de nível terciário, visando aperfeiçoar o tempo de atendimento e priorizar condições graves, tem sido implantada a classificação de risco baseada no Sistema de Triagem de Manchester (STM). Criado em 1997 no Reino Unido, o STM trabalha com fluxogramas de acordo com a condição clínica do paciente, sendo cinco as classificações principais distribuídas em cores: emergência (vermelho), muito urgente (laranja), urgência (amarelo), pouco urgente (verde) e não urgente (azul), critérios esses, passíveis de reavaliação e reclassificação ${ }^{4-6}$.

A possibilidade de que esteja ocorrendo uso inadequado do componente hospitalar de atenção às urgências, pode ser averiguada por meio da aplicação de um indicador indireto de qualidade da atenção primária, denominado Internações por Condições Sensíveis a Atenção Primária (ICSAP). Trata-se de uma listagem de diagnósticos e morbidades que podem ser atendidas oportuna e efetivamente pela atenção primária, sem necessidade de hospitalização. As taxas de ICSAP refletem o acesso, a cobertura, a qualidade e o desempenho da atenção primária e podem explicar a sobrecarga existente em outros pontos da rede, principalmente nas unidades de pronto-socorro ${ }^{7}$. Assim, este estudo teve por objetivo conhecer o perfil e o tempo de permanência dos usuários admitidos no pronto-socorro adulto.

\section{MÉTODO}

Trata-se de uma pesquisa transversal com delineamento descritivo e abordagem quantitativa do tipo analítico, realizado por meio da busca ativa de informações nos prontuários sobre atendimentos ocorridos no pronto-socorro adulto (PSA), de um hospital público de ensino na cidade de Uberaba-MG.

A instituição faz parte da rede de hospitais universitários federais e é considerada de grande porte, tida como referência de alta complexidade para 27 municípios da macrorregião do Triângulo Sul de Minas Gerais. Atualmente, conta com 292 leitos de internação ativos. 0 pronto-socorro adulto é a principal porta de entrada do serviço e conta com 22 leitos de internação, estando esses distribuídos entre três enfermarias e um quarto de isolamento ${ }^{8}$. Segundo dados internos do serviço, a unidade teve uma média de ocupação de 38,18 usuários/dia, 450 internações/mês e aproximadamente 1.100 atendimentos/mês, no ano de 20189. 0 acesso dos usuários ao PSA ocorre de duas maneiras: mediante regulação online, por meio da utilização de um sistema de informação pertencente ao Ministério da Saúde, denominado SISREG II, ou diretamente da rede municipal de atendimento pré-hospitalar, quando se trata de situações de urgência e emergência.

A amostra foi escolhida por conveniência. Foram incluídos na pesquisa todos os usuários maiores de 18 anos, que se encontravam internados no PSA no período de realização da coleta 
dos dados, realizada entre novembro de 2017 e janeiro de 2018. Foram excluídos os usuários que estavam em atendimento na unidade, na modalidade de observação.

Os dados foram analisados a partir do programa Statistical Package Social Science (SPSS) versão 17.0 para Windows. Anteriormente os dados sofreram o processo de dupla digitação para análise e reforço interno da consistência. A variável dependente utilizada, foi o tempo de permanência do paciente no PSA, dividido nas categorias maior do que 24 horas ou menor do que 24 horas. As variáveis independentes foram: sexo, idade, cidade de origem, unidade que realizou a transferência, local de admissão, clínica responsável, Classificação Internacional de Doenças e Problemas Relacionados à Saúde (CID-10), classificação de risco segundo o Sistema de Triagem de Manchester e tempo de espera por atendimento.

O agrupamento das variáveis foi realizado da seguinte forma: sexo (masculino e feminino) e idade (18 a 59 anos e maiores de 60 anos). Quanto à cidade de origem, dividiu-se entre Uberaba e outras, uma vez que existem 27 municípios da macrorregião que são atendidos pela instituição. As unidades de origem foram categorizadas em serviço de atendimento préhospitalar móvel e fixo, representados respectivamente pelo Serviço de Atendimento Móvel de Urgência (SAMU) e pelas Unidades de Pronto Atendimento (UPA); unidades inter-hospitalares municipais e intermunicipais; demanda espontânea e ambulatório de especialidades vinculado à instituição.

Os locais de admissão no pronto-socorro foram categorizados em sala de emergência e corredor. As especialidades médicas responsáveis pelo atendimento, foram divididas entre clínica médica e cirúrgica/ortopedia. Quanto aos diagnósticos, esses foram divididos, segundo a CID-10, em dois grupos: os que pertencem à lista de ICSAP e os que pertencem às condições consideradas "não sensíveis", por não fazerem parte da lista de ICSAP. A classificação de risco foi dividida entre as categorias: urgente (cor vermelha), emergente (cor laranja) e outras (cores amarelo, verde e azul). O tempo de espera por atendimento, correspondente ao período compreendido, entre o horário de inclusão do usuário no sistema hospitalar, denominado AGHU, e o horário início do atendimento registrado no relatório de enfermagem, foi dividido em: até 10 minutos, de 11 a 60 minutos, mais de 60 minutos, em conformidade com o STM.

Utilizou-se análise estatística descritiva para obtenção de frequência absoluta e relativa e, posteriormente foi realizado o modelo de regressão logística binária, por meio da análise bruta, que testou as variáveis independentes e sua associação com a variável dependente, com adoção do valor de $\mathrm{p}<0,20$, para proceder à análise ajustada.

O projeto foi aprovado pelo Comitê de Ética e Pesquisa (CEP), da Universidade Federal do Triângulo Mineiro, Parecer número 1.781.682.

\section{RESULTADOS}

Foram considerados 304 pessoas internadas. Em relação ao tempo de permanência no pronto-socorro adulto, a maioria (74\%) dos usuários permaneceu mais do que 24 horas no setor (Tabela 1).

A maioria era do sexo masculino, com idade entre 18 a 59 anos, procedentes do município de Uberaba-MG. Deram entrada no serviço por intermédio do transporte vinculado ao atendimento pré-hospitalar do município, em sua maioria admitidos na sala de emergência, tendo como responsável pelo atendimento a clínica médica (Tabela 1).

Quanto à classificação de risco, a maioria foi classificada na categoria "outros", correspondente a um menor grau de prioridade (cores amarelo, verde, azul ou branco), e o tempo de espera para o início de atendimento, foi de até 10 minutos (Tabela 1). A maioria dos atendimentos não foram sensíveis à Atenção Primária a Saúde (APS). 
Tabela 1. Características sociodemográficas e de atendimento, de acordo com o tempo de permanência no pronto-socorro adulto. Uberaba, novembro de 2017 a janeiro de 2018.

\begin{tabular}{|c|c|c|}
\hline Variáveis & Total & $(\%)$ \\
\hline \multicolumn{3}{|l|}{ Tempo de permanência } \\
\hline Até 24 horas & 79 & 26,0 \\
\hline$>24$ horas & 225 & 74,0 \\
\hline \multicolumn{3}{|l|}{ Sexo } \\
\hline Feminino & 111 & 36,5 \\
\hline Masculino & 193 & 63,5 \\
\hline \multicolumn{3}{|l|}{ Clínica Responsável } \\
\hline Médica & 180 & 59,2 \\
\hline Cirúrgica/Ortopedia & 124 & 40,8 \\
\hline \multicolumn{3}{|l|}{ Cidade } \\
\hline Uberaba & 190 & 62,5 \\
\hline Outras & 114 & 37,5 \\
\hline \multicolumn{3}{|l|}{ Idade } \\
\hline 18 a 59 anos & 169 & 55,6 \\
\hline$>60$ anos & 135 & 44,4 \\
\hline \multicolumn{3}{|l|}{ Unidade que referenciou } \\
\hline Pré-hospitalar & 129 & 42,4 \\
\hline Transferência intermunicipal & 73 & 24,0 \\
\hline Demanda espontânea & 55 & 18,1 \\
\hline Ambulatório de especialidades & 47 & 15,5 \\
\hline \multicolumn{3}{|l|}{ STM } \\
\hline Urgente e Emergente & 118 & 38,8 \\
\hline Outros & 186 & 61,2 \\
\hline \multicolumn{3}{|l|}{ Tempo espera por atendimento } \\
\hline Até $10 \mathrm{~min}$ & 172 & 56,6 \\
\hline 11 a $60 \mathrm{~min}$ & 52 & 17,1 \\
\hline $60 \mathrm{~min}$ & 80 & 26,3 \\
\hline \multicolumn{3}{|l|}{ Local de admissão } \\
\hline Sala de emergência & 171 & 56,3 \\
\hline Corredor & 133 & 43,8 \\
\hline \multicolumn{3}{|l|}{ CID-10 } \\
\hline ICSAP & 72 & 23,7 \\
\hline Outros (não sensível à APS) & 232 & 76,3 \\
\hline
\end{tabular}

Na análise bruta, sujeitos atendidos pela clínica médica (OR=3,04; IC95\%= 1,78-5,15), referenciados pelo pré-hospitalar $(\mathrm{OR}=3,99$; IC95\%= 1,84-8,19), por demanda espontânea (OR=4,31; IC95\%: 1,77-10,53) e por transferência intermunicipal (OR=2,37; IC95\%: 1,10-5,09), admitidos na sala de emergência (OR=1,46; IC95\%: 0,87-2,44) e classificados quanto ao CID por condições não sensíveis à atenção primária (OR=1,75; IC95\%: 0,99-3,10) apresentaram associação significativa com o tempo de permanência no pronto-socorro adulto acima de 24 horas (Tabela 2).

$\mathrm{Na}$ análise ajustada, mantiveram-se associados ao tempo de permanência os usuários atendidos pela clínica médica $(\mathrm{OR}=2,97$; IC95\%: 1,69-5,20), referenciados ao serviço pelo préhospitalar (OR=4,27; IC95\%: 2,00-9,11), transferência intermunicipal (OR=3,04; IC95\%: 1,346,85) ou vindos por demanda espontânea (OR=4,57; IC95\%:1,79-11,60), além daqueles admitidos na sala de emergência (OR=1,22; IC95\%: 0,67-2,15), e classificados no CID-10 em outros grupos que não se enquadram na lista de condições sensíveis à atenção primária (OR=2,02; IC95\%: 1,09-3,75) (Tabela 2).

Os resultados demonstraram que os sujeitos atendidos pela clínica médica referenciados por demanda espontânea tem quatro vezes mais chance de permanecer internados por mais de 24 horas no serviço. 
Tabela 2. Razão de prevalência bruta e ajustada entre tempo de permanência no prontosocorro adulto e características demográficas e de atendimento. Uberaba, novembro de 2017 a janeiro de 2018.

\begin{tabular}{|c|c|c|c|c|}
\hline Variáveis & $\begin{array}{l}\text { Análise bruta } \\
\text { OR (IC 95\%) }\end{array}$ & $\mathbf{p}^{\mathbf{a}}$ & $\begin{array}{l}\text { Análise ajustada } \\
\text { OR (IC 95\%) }\end{array}$ & $\mathbf{p}^{\mathbf{b}}$ \\
\hline Sexo & & 0,818 & & \\
\hline Masculino & 1 & & & \\
\hline Feminino & $1,06(0,62-1,82)$ & & & \\
\hline Cidade & & 0,237 & & \\
\hline Outras & 1 & & & \\
\hline Uberaba & $1,41(0,84-2,37)$ & & & \\
\hline Clinica responsável & & $<0,001$ & & $<0,001$ \\
\hline Cirúrgica/ortopedia & 1 & & 1 & \\
\hline Médica & $3,04(1,78-5,15)$ & & $2,97(1,69-5,20)$ & \\
\hline Idade & & 0,809 & & \\
\hline$>60$ anos & 1 & & & \\
\hline 18 a 59 anos & $1,06(0,64-1,78)$ & & & \\
\hline Unidade de referência & & $<0,001$ & & 0,001 \\
\hline Ambulatório de especialidades & 1 & & 1 & \\
\hline Pré-hospitalar & $3,99(1,84-8,19)$ & & $4,27(2,00-9,11)$ & \\
\hline Transferência intermunicipal & $2,37(1,10-5,09)$ & & $3,04(1,34-6,85)$ & \\
\hline Demanda espontânea & $4,31(1,77-10,53)$ & & $4,57(1,79-11,60)$ & \\
\hline STM & & 0,655 & & \\
\hline Outros & 1 & & & \\
\hline Emergente e urgente & $1,13(0,66-1,91)$ & & & \\
\hline Tempo de espera por atendimento & & 0,392 & & \\
\hline$>60 \mathrm{~min}$ & 1 & & & \\
\hline $11-60 \mathrm{~min}$ & $1,69(0,75-3,83)$ & & & \\
\hline Até $10 \mathrm{~min}$ & $1,36(0,76-2,45)$ & & & \\
\hline Local de admissão & & 0,152 & & 0,486 \\
\hline Corredor & 1 & & 1 & \\
\hline Sala de emergência & $1,46(0,87-2,44)$ & & $1,22(0,67-2,15)$ & \\
\hline CID-10 & & 0,055 & & 0,026 \\
\hline ICSAP & 1 & & 1 & \\
\hline Outros (não sensível à APS) & $1,75(0,99-3,10)$ & & $2,02(1,09-3,75)$ & \\
\hline
\end{tabular}

\section{DISCUSSÃO}

A maioria dos usuários era do sexo masculino. Os atendimentos eram realizados em sua maioria mediante regulação assistencial, pela qual observa-se predominância de quadros clínicos considerados de menor risco de acordo com o STM, já que a estabilidade hemodinâmica é um dos critérios para o transporte médico-hospitalar. Outros estudos também comprovaram o frequente atendimento aos homens nas unidades de pronto-socorro, principalmente, associado às causas traumáticas, tendo em vista uma maior relação do homem com acidentes de trânsito e a violência urbana, elevando as taxas de óbito5,10,11.

A clínica médica foi responsável pelo maior número de atendimentos, resultado que corrobora com o estudo realizado em um pronto-socorro adulto, na cidade de Belo Horizonte $(\mathrm{MG})^{10}$. Esse achado, pode ter relação com a elevada prevalência e a agudização de doenças crônicas não transmissíveis.

0 tempo de permanência dos usuários foi maior do que 24 horas, resultado que difere de outros trabalhos, na qual a maioria dos usuários teve como destino principal a alta hospitalar4,13,14. Em relação aos pacientes atendidos pela clínica médica, que foram maioria, estes apresentaram um maior tempo de internação. Um estudo realizado na cidade de São Paulo também encontrou dados semelhantes entre atendimento de clínica médica associado ao maior tempo de internação ${ }^{13}$. A unidade em questão, é referência no atendimento de alta complexidade, e admite majoritariamente usuários graves, que necessitam de investigação 
clínica para estabelecer condutas, o que pode ocasionar demora na transferência intrahospitalar.

$\mathrm{Na}$ classificação de risco, de acordo com o STM, predominaram as cores amarelo, verde e azul, consideradas com menor prioridade de atendimento. Dois estudos na cidade de Belo Horizonte (MG), sendo um deles classificado como hospital de grande porte, mostraram a classificação por nível de prioridade predominante nas cores amarelo e verde, dados semelhantes aos deste estudo 4,11 .

Investigação que analisou a demanda atendida em uma unidade de emergência também demonstrou alta frequência de casos classificados, como verde e azul, e citou a falta de tecnologias e recursos humanos nos níveis primário e secundário, efetividade dos procedimentos disponíveis, bem como, a valorização de serviços de urgência / emergência como fatores contribuintes para o excesso de demanda nas unidades de pronto-socorro 5 .

Estudo realizado em Portugal avaliou o impacto de doenças crônicas nas condições sensíveis à atenção primária e encontrou uma maior prevalência $(58,3 \%)$ das hospitalizações por condições não sensíveis à APS, sendo que dessas, a maior parte foi atendida por especialidade de clínica médica $(97,6 \%)$ e admitidas em caráter de emergência $(87,2 \%)^{16}$.

0 percentual de internações por condições sensíveis à atenção primária (ICSAP), identificado pela presente pesquisa, está em consonância com trabalho realizado em grandes municípios do Brasil, que avaliou a evolução das ICSAP, e encontrou um declínio na proporção dessas em aproximadamente $15 \%$, o que pode ser explicado pelo crescimento de cobertura das Estratégia de Saúde da Família (ESFs), nos diferentes contextos dos municípios brasileiros ${ }^{15}$.

\section{CONCLUSÃO}

Em sua maioria, os pacientes internados foram do sexo masculino, com patologias clínicas, procedentes do atendimento pré-hospitalar do próprio município, atendidos pela equipe da clínica médica, com quadros clínicos considerados de menor risco de acordo com o STM.

Em síntese os resultados deste estudo sugerem que houve poucas internações por condições sensíveis à atenção primária e pessoas referenciados por demanda espontânea tem 4 vezes mais chance de permanecer internadas por mais de 24 horas no serviço.

O fato de ser o único hospital da Macrorregião de Saúde Triângulo Sul, credenciado em alta complexidade, pelo Sistema Único de Saúde (SUS), gera uma demanda por internação independente da disponibilidade de leitos, levando a internações nos corredores, saturação da capacidade operacional e permanência de usuários na unidade por período superior a 24 horas.

Por se tratar de um estudo prospectivo, a dificuldade de acesso aos prontuários médicos, foi a principal limitação, levando à morosidade na coleta de dados. Até o momento da realização do estudo, a instituição não dispunha de prontuário eletrônico.

Faz-se necessário, pesquisas na própria instituição, em maior temporalidade e em associação com os outros pontos de atenção da rede municipal e macrorregional de saúde, quanto ao fluxo e a demanda dos serviços, possibilitando a identificação das fragilidades da rede e a adoção de intervenções que possam impactar positivamente na redução do tempo de permanência e da saturação da capacidade operacional.

\section{REFERÊNCIAS}

1. Moraes DS, Cordeiro NM, Fonseca ADG, Silva CSO, Souza LPS, Lopes JR. Fatores associados à internação prolongada nas admissões pela urgência e emergência. Rev Univ Vale Rio Verde [Internet]. 2017 [citado em 20 jul 2020]; 15(2):680-91. Disponível em: http://periodiscos.unincor.br/index.php/revistaunincor/article/view/3770. DOI: http://dx.d oi.org/10.5892/ruvrd.v15i2.3770

2. Martins TA, Santos CB, Santos MA, Rodrigues FFL, Pedersoli CE, Zanetti ML. Demanda de usuários com diabetes mellitus em unidade de urgência de um hospital universitário. Ciênc 
Cuid Saúde [Internet]. 2016 [citado em 17 jun 2020]; 15(2):312-20. Disponível em: www.researchgate.net/publication/309594964.

DOI: https://doi.org/10.4025/cienccuidsaude.v15i2.28272

3. Casarolli ACG, Eberhardt TD, Nicola AL, Fernandes LM. Nível de complexidade assistencial e dimensionamento de enfermagem no pronto-socorro adulto de um hospital público. Rev Enferm UFSM [Internet]. 2015 [citado em 20 jul 2020]; 5(2):278-85. Disponível em: https://periodicos.ufsm.br/reufsm/article/view/16811.

https://doi.org/10.5902/2179769216811

4. Gonçales PC, Pinto Júnior D, Salgado PO, Chianca TCM. Relationship between risks tratification, mortality and length of stay in a Emergency Hospital. Invest Educ Enferm. [Internet]. 2015 [citado em 14 mar 2020]; 33(3):424-31. Disponível em: www.scielo.org.co/scielo.php?script=sci_arttext\&pid=S0120-53072015000300005. DOI: https://doi.org/10.17533/udea.iee.v33n3a05

5. Feijó VBER, Cordoni Junior L, Souza RKT, Dias AO. Análise da demanda atendida em unidade de urgência com classificação de risco. Saúde Debate [Internet]. 2015 [citado em 14 mar 2020]; 39(106):627-36. Disponível

em: https://www.scielo.br/j/sdeb/a/9rrjMmGS8Hxdv4ZRVDFPcTn/?lang=pt\&format=pdf. DOI: https://doi.org/10.1590/0103-110420151060003005

6. Anziliero F, Dal Soler BE, Silva BA, Tanccini T, Beghetto MG. Sistema Manchester: tempo empregado na classificação de risco e prioridade para atendimento em uma emergência. Rev Gaúch Enferm. [Internet]. 2016 [citado em 22 ago 2020]; 37(4):e64753. Disponível em: https://www.scielo.br/j/rgenf/a/ZPt8CVtgXpftkT7MszL8KtP/abstract/?lang=pt.

DOI: https://doi.org/10.1590/1983-1447.2016.04.64753

7. Alfradique ME, Bobolo PF, Dourado I, Lima-Costa MF, Macinko J, Mendonça CS, et al. Internações por condições sensíveis à atenção primária: a construção da lista brasileira como ferramenta para medir o desempenho do sistema de saúde (Projeto ICSAP - Brasil). Cad Saúde Pública [Internet]. 2009 [citado em 20 mar 2020]; 25(6):1337-49. Disponível em: https://www.scielo.br/j/csp/a/y5n975h7b3yW6ybnk6hJwft/abstract/?lang=pt.

DOI: https://doi.org/10.1590/S0102-311X2009000600016

8. Brasil. Empresa Brasileira de Serviços Hospitalares. Institucional: Nossa História [Internet]. Uberaba-MG, 2020 [citado em 31 nov 2020]. Disponível em: https://www.gov.br/ebserh/pt$\mathrm{br} /$ hospitais-universitarios/regiao-sudeste/hc-uftm/acesso-a-informacao/institucional

9. Universidade Federal do Triângulo Mineiro. Relatório Mensal de Indicadores Assistenciais. Uberaba-MG, 2019 [citado em 28 nov 2019]. Disponível em: https://www.gov.br/ebserh/pt-br/hospitais-universitarios/regiao-sudeste/hc-uftm 10. Guedes HM, Martins JCA, Chianca TCM. Valor de predição do Sistema de Triagem de Manchester: avaliação dos desfechos clínicos de usuários. Rev Bras Enferm. [Internet]. 2015 [citado em 20 mar 2020]; 68(1):45-51. Disponível em: https://www.scielo.br/j/reben/a/dmdJd5CB9bhxfJ7QTVcgXVf/?lang=pt. DOI: https://doi.org/10.1590/0034-7167.2015680107p

11. Silva ADC, Chianca TCM, Pádua DR, Guimarães GL, Correa AR. Caracterização dos atendimentos de um pronto-socorro adulto público segundo o sistema de triagem de Manchester. Rev Min Enferm. [Internet]. 2019 [citado em 17 jun 2020]; 23:1-8. Disponível em: https://cdn.publisher.gn1.link/reme.org.br/pdf/1178.pdf.

DOI: https://doi.org/10.5935/1415-2762.20190026

12. Pinto Júnior D, Salgado PO, Chianca TCM. Validade preditiva do Protocolo de Classificação de Risco de Manchester: avaliação da evolução dos usuários admitidos em um pronto atendimento. Rev Latinoam Enferm. [Internet]. 2012 [citado em 20 mar 2020]; 20(6):1041-7. Disponível

em: https://www.scielo.br/j/rlae/a/zhQNMVSnMr53scQRVJ7jNYm/?lang=pt\&format=pdf. DOI: https://doi.org/10.1590/S0104-11692012000600005 
13. Ribeiro RM, Cesarino CB, Ribeiro RCHM, Rodrigues CC, Bertolin DC, Pinto MH, et al. Caracterização do perfil das emergências clínicas no pronto-atendimento de um hospital de ensino. Rev Min Enferm. [Internet]. 2014 [citado em 21 jul 2020]; 18(3):533-44. Disponível em: https://www.reme.org.br/artigo/detalhes/944. DOI: http://www.dx.doi.org/10.5935/14152762.20140039

14. Becker JB, Lopes MCBT, Pinto MF, Campanharo CRV, Barbosa DA, Batista REA. Triagem no Serviço de Emergência: associação entre as suas categorias e os desfechos do paciente. Rev Esc Enferm USP [Internet]. 2015 [citado em 20 mar 2021]; 49(5):783-9. DOI: http://www.dx.doi.org/10.1590/S0080-623420150000500011

15. Castro ALB, Andrade CLT, Machado CV, Lima LD. Condições socioeconômicas, oferta de médicos e internações por condições sensíveis à atenção primária em grandes municípios do Brasil. Cad Saúde Pública [Internet]. 2015 [citado em 21 jul 2021]; 31(11):2353-66. Disponível em: https://www.scielo.br/j/csp/a/jSv9TzXPgjWTvFJzbZb9cVq/abstract/?lang=pt. DOI: https://doi.org/10.1590/0102-311X00126114

16. Dantas I, Santana R, Sarmento J, Aguiar P. The impact of multiple chronic diseases on hospitalizations for ambulatory caresensitive conditions. BMC Health Serv Res. [Internet]. 2016 [citado em 20 mar 2021]; 16(1):348. Disponível em: https://bmchealthservres.biomedcentral.com/articles/10.1186/s12913-016-1584-2.pdf. DOI: https://doi.org/10.1186/s12913-016-1584-2

Editora Associada: Vania Del Arco Paschoal

\section{CONTRIBUIÇÕES}

Cintia Machado Dutra, Lúcia Aparecida Ferreira e Suzel Regina Ribeiro Chavaglia contribuíram na concepção, redação e revisão. Lorena Daher e Joyce Caroline Lucas Santos participaram da concepção, coleta e análise dos dados. Débora de Oliveira Sacramento atuou na redação.

\section{Como citar este artigo (Vancouver)}

Dutra CM, Daher L, Chavaglia SRR, Ferreira LA, Sacramento DO, Santos JCL. Perfil e tempo de permanência dos usuários admitidos no pronto-socorro adulto de um hospital público. REFACS [Internet]. 2021 [citado em inserir dia, mês e ano de acesso]; 9(4):953-60. Disponível em: inserir link de acesso. DOI: inserir link do DOI.

\section{Como citar este artigo (ABNT)}

DUTRA, C. M.; DAHER, L.; CHAVAGLIA, S. R. R.; FERREIRA, L. A.; SACRAMENTO, D. 0.; SANTOS, J. C. L. Perfil e tempo de permanência dos usuários admitidos no pronto-socorro adulto de um hospital público. REFACS, Uberaba, MG, v. 9, n. 4, p. 953-60, 2021. Disponível em: inserir link de acesso. Acesso em: inserir dia, mês e ano de acesso. DOI: inserir link do DOI.

\section{Como citar este artigo (APA)}

Dutra, C.M., Daher, L., Chavaglia, S.R.R., Ferreira, L.A., Sacramento, D.O., \& Santos J.C.L. (2021). Perfil e tempo de permanência dos usuários admitidos no pronto-socorro adulto de um hospital público. REFACS, 9(4), 953-60. Recuperado em: inserir dia, mês e ano de acesso de inserir link de acesso. DOI: inserir link do DOI. 